\title{
¿La demanda y la rentabilidad estimulan la acumulación de capital? Un análisis para el Brasil
}

\author{
Henrique Morrone
}

RESUMEN

El objetivo de este artículo consiste en probar si la participación de los beneficios en el producto y la capacidad utilizada afectan (causan en el sentido de Granger) a la acumulación de capital en el Brasil durante el período 1950-2008. Se emplea la metodología desarrollada por Toda y Yamamoto (1995) para verificar la hipótesis de no causalidad de Granger. Los resultados indican que la capacidad utilizada causa, en el sentido de Granger, la acumulación de capital en la economía brasileña y, por otra parte, que la participación de los beneficios en el producto no causa, en el sentido de Granger, la razón inversión-capital nacional. Esto corrobora la propuesta kaleckiana centrada en el papel fundamental del acelerador y sugiere que la economía del Brasil puede crecer con concentración o desconcentración del ingreso, siempre que haya un arreglo institucional adecuado.

PALABRAS CLAVE

CLASIFICACIÓN JEL

AUTOR
Desarrollo económico, formación de capital, crecimiento económico, modelos econométricos, Brasil

$\mathrm{O} 1, \mathrm{~B} 5, \mathrm{C} 1$

Henrique Morrone es profesor adjunto de la Universidad Federal de Rio Grande do Sul (Ufrgs), Brasil. hmorrone@hotmail.com 


\section{I}

\section{Introducción}

El débil desempeño económico es un problema que afecta a muchos países. Un bajo nivel de actividad económica puede conducir a la economía a un círculo vicioso de modesta productividad. La reducción de la demanda efectiva es uno de los factores que explican el desempeño económico mediocre de un grupo de países. Por otra parte, un crecimiento pronunciado de la demanda puede estimular la producción en sectores dinámicos y fomentar economías de escala, el aumento de la productividad y la expansión económica.

En ese sentido, la redistribución del ingreso en favor de los trabajadores puede incentivar la demanda y la actividad de la economía en países con grandes mercados internos. La redistribución puede engrosar dicho mercado interno e incentivar de ese modo considerables economías de escala. Además, si la redistribución del ingreso hacia los trabajadores produce mejoras en materia de educación y salud, estas tendrán efectos positivos en la productividad del trabajo y el crecimiento económico a largo plazo. En algunos países, esto puede incluso reducir la inestabilidad política, en beneficio de las reformas institucionales y la provisión de bienes públicos.

Por esas razones, es de vital importancia examinar el régimen de crecimiento de la economía brasileña para establecer si la redistribución del ingreso en favor de los trabajadores produce la expansión del producto en la economía. Si el régimen de crecimiento se basa en los salarios (wage-led), la redistribución de ingresos surtirá efectos positivos. Por otra parte, si el régimen de la demanda sigue una trayectoria basada en las ganancias (profit-led), la redistribución producirá la contracción de la actividad económica.

El principal objetivo de este trabajo consiste en verificar si la participación de los beneficios en el producto y la capacidad utilizada causan, en el sentido de Granger, la acumulación de capital de la economía brasileña en el período 1950-2008. En otras palabras, se intenta comprobar si las variaciones de la relación beneficios-producto y de la capacidad utilizada preceden en el tiempo a las variaciones de la acumulación. Sobre la base de las ideas de Kalecki (1971) y Steindl (1952), se examinará la validez de la función de inversión para el caso brasileño y, en consecuencia, se determinará el régimen de expansión de la economía (basado en los salarios o en las ganancias). Con respecto al período de análisis, se utilizó una muestra de datos tomados de IPEA (2011) y Marquetti (2000) de 1950 a 2008. Se empleó la base de datos del capital nacional de este autor extendida hasta 2008. Asimismo, se recurrió a la técnica desarrollada por Toda y Yamamoto (1995) para probar la causalidad entre las series temporales de las variables: acumulación de capital, relación beneficios-producto, y capacidad utilizada.

El presente artículo se divide en cinco secciones además de esta Introducción. Mientras que en la segunda sección se presenta una breve retrospectiva del desempeño reciente de la economía brasileña, en la tercera se examina la relación entre distribución y actividad económica. En la cuarta sección se describe la metodología empleada y en la quinta se detallan los resultados. Por último, en la sexta sección se presentan las conclusiones.

\section{II}

\section{La economía brasileña en la década de 2000: breve retrospectiva}

Desde mediados de la década de 2000 se observa un marcado crecimiento de la economía brasileña. Entre 2004 y 2010, el producto interno bruto (PIB) aumentó un $4 \%$ anual y el PIB per cápita un 2,8\% al año, a pesar de la crisis financiera internacional que afectó al país a partir del cuarto trimestre de 2008. Ese desempeño prácticamente duplica el registrado entre 1980 y 2003, cuando la economía creció a una tasa media del 2,2\% anual. Cabe destacar que el Brasil, que integraba el grupo de países con altas tasas de crecimiento durante la edad de oro del desarrollo capitalista (1950-1973) — cuando el PIB se expandió a una tasa anual del 7\%—, 
pasó a formar parte del grupo con ritmos de crecimiento menores durante el capitalismo neoliberal (1980-2007). Sin embargo, pese a la reanudación de cierto dinamismo, el país creció a un ritmo inferior con respecto al período 1950-1973 y a las economías en desarrollo entre los años 2004 y 2010 (Naciones Unidas, 2010).

Un breve resumen de las razones de la recuperación permite entender los cambios en la conducción de la política económica con relación al período anterior y ofrece elementos relevantes para los análisis realizados en este trabajo.

El panorama internacional prevaleciente hasta 2008 contribuyó a los resultados observados en el Brasil. La economía mundial creció rápidamente, impulsada por China y la India. En los primeros años de la década de 2000, la economía brasileña se benefició con el aumento de las exportaciones y los precios internacionales muy favorables. La deuda externa neta al final del segundo semestre de 2007 era de 49.300 millones de dólares, un valor nominal inferior al vigente en 1980. La vulnerabilidad ante las crisis internacionales disminuyó y el país se convirtió en un gran receptor de inversión directa neta. La crisis internacional se produjo cuando el Brasil contaba con un gran volumen de reservas y capacidad para aplicar políticas anticíclicas.

Un aspecto clave de la expansión fue la decisión de implementar un programa de desarrollo basado en el mercado interno. $\mathrm{Al}$ respecto se tomaron tres medidas fundamentales. La primera fue la decisión del Gobierno brasileño de adoptar un plan orientado al desarrollo económico. El Plan de Aceleración del Crecimiento (PAC) representó la recuperación del papel del Estado en la planificación económica y la coordinación de las inversiones públicas y de las empresas estatales y privadas. Como se observa en el gráfico 1, la tasa de inversión se recuperó rápidamente después de la adopción del PAC, pasando del 15,9\% en 2005 al 19,5\% en 2010 .

La segunda fue la implementación de políticas redistributivas, como el programa Bolsa Familia y el aumento real del salario mínimo, que produjeron importantes efectos en el crecimiento del consumo de las familias y el incremento de la actividad económica. En el gráfico 2 se aprecia la evolución de los gastos sociales con respecto al PIB en el período 1994-2008. Se observa un cambio en la trayectoria de la política económica a lo largo de la década de 2000. Una de las consecuencias de la redistribución fue el mejoramiento de los indicadores de distribución del ingreso. El coeficiente de Gini, que mide el grado de desigualdad de la economía, pasó de 0,61 en 1990 a 0,54 en 2009 (IPEA, 2011) indicando la reducción de la desigualdad en los ingresos de los trabajadores.

La tercera medida fue la ampliación de la oferta de crédito en los más diversos segmentos del mercado financiero, encabezada sobre todo por los bancos públicos, que desempeñaron el papel de liderazgo en el proceso de expansión crediticia. Con el aumento de la oferta de crédito de los bancos públicos y privados, la cantidad de crédito con respecto al PIB se acrecentó considerablemente de 2000 a 2010.

GRÁFICO 1



Fuente: elaboración propia sobre la base de Instituto Brasileño de Geografía y Estadística (IBGE), "Tabela de recursos e usos", 2011 [en línea] http://www.ibge.gov.br/home/estatistica/economia/contasnacionais/2011. 
GRÁFICO 2

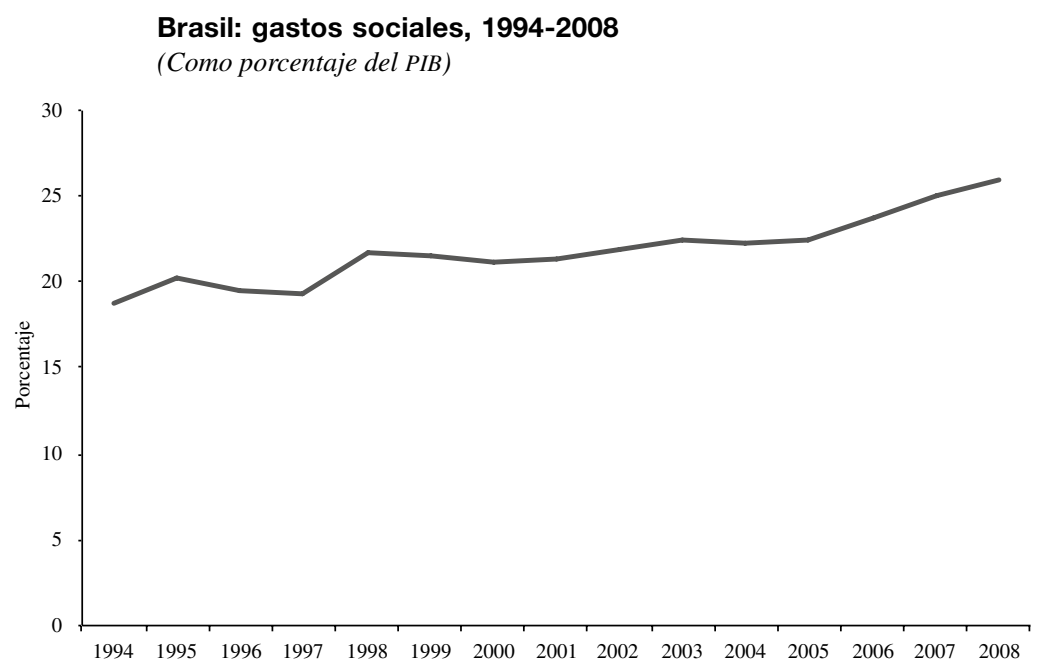

Fuente: elaboración propia sobre la base de Instituto Brasileño de Geografía y Estadística (IBGE), “Tabela de recursos e usos”, 2011 [en línea] http://www.ibge.gov.br/home/estatistica/economia/contasnacionais/2011, y Comisión Económica para América Latina y el Caribe (CEPAL).

Cabe destacar que uno de los resultados de la política de expansión por la vía del mercado interno fue la reducción de la tasa de desempleo, que pasó del $10,5 \%$ en diciembre de 2002 a un 5,3\% en diciembre de 2010. Asimismo, el incremento de los empleos formales fue fundamental para respaldar desde el punto de vista político y económico el conjunto de medidas adoptadas por el Estado brasileño.

La reducción de la desigualdad y el mejoramiento de las condiciones de vida de la población desfavorecida pueden estimular el crecimiento. En primer lugar, porque mejoran la alimentación de las personas desde el punto de vista de la calidad y la cantidad, lo que se traduce en ganancias de productividad del trabajo. La redistribución del ingreso reduce la pobreza, con efectos positivos en la salud y la educación (Deaton, 2003), que aumentan la productividad laboral a mediano y largo plazo. Además, en las economías igualitarias se logra con mayor facilidad el consenso para ejecutar reformas y esto a su vez reduce la inestabilidad política.

El objetivo de la política monetaria a lo largo del período analizado fue el control de la inflación, excepto durante el punto culminante de la crisis financiera internacional entre fines de 2008 y el primer semestre de 2009. La elevada tasa de interés tiene repercusiones en la política fiscal y en el costo de financiamiento de la deuda pública. La disminución de las tasas de interés reales y nominales permitiría el cambio en la composición de los gastos públicos, con la reducción de los gastos destinados al pago de intereses y la posibilidad de aumentar las inversiones y los gastos públicos, y aminorar los impuestos.

Otro efecto relevante se produce en el tipo de cambio. La diferencia entre la tasa de interés de los títulos de la deuda pública brasileña en relación con las tasas internacionales fue un importante factor de atracción de recursos financieros y, en consecuencia, de apreciación del real. El tipo de cambio sobrevaluado produce dos efectos perversos en el crecimiento económico. En primer lugar, aumenta el déficit en cuenta corriente y la necesidad de atraer ahorro externo a través de la cuenta de capital, lo que se traduce en el incremento del pasivo externo. En segundo lugar, reduce la competitividad de la industria, acelerando el proceso de desindustrialización a mediano y largo plazo. Sin embargo, la apreciación cambiaria facilita la adquisición de maquinaria y equipos para el sector industrial, lo que podría traducirse en un aumento de la productividad.

De hecho, el tipo de cambio apreciado evita un mayor incremento de los precios, con efectos positivos en el salario real y, por ende, en el consumo. Esto puede actuar como un factor de estímulo al crecimiento en las grandes economías, que poseen un amplio mercado interno. Así, el efecto neto de la sobrevaluación cambiaria en la economía depende de las especificidades de cada región y puede ser expansivo o contractivo.

En el cuadro 1 se resume el crecimiento brasileño entre 1989 y 2008, observándose una expansión de la tasa de crecimiento del PIB después de 2003. 
Brasil: tasa de crecimiento del producto interno bruto (PIB), 1989-2008 (En porcentajes)

\begin{tabular}{lcc}
\hline Período & PIB & Capital nacional neto \\
\hline $1989-2003$ & 2,21 & 2,63 \\
$1989-1997$ & 2,67 & 2,98 \\
$1997-2003$ & 1,60 & 2,16 \\
$2003-2008$ & 4,70 & 3,02 \\
\hline
\end{tabular}

Fuente: elaboración propia.

\section{III}

\section{Desigualdad de ingresos y actividad económica}

El desarrollo económico es el resultado de profundos cambios estructurales en la dirección de actividades generadoras de mayor valor agregado, poseedoras de economías de escala dinámicas ${ }^{1}$. La interacción entre oferta y demanda y la distribución de los beneficios del proceso de expansión del excedente productivo desempeñan un papel relevante en la explicación del desarrollo. Así, el examen de algunos aspectos de la relación entre la distribución del ingreso y el nivel de actividad económica es crucial para entender mejor el proceso de expansión de las economías.

En la literatura económica se ha investigado constantemente si la redistribución del ingreso en favor de los trabajadores puede inducir el aumento del nivel de actividad económica. Los resultados empíricos y teóricos son variados y destacan la complejidad de la relación entre las dos variables.

En algunos estudios, entre ellos los de Berni, Marquetti y Kloeckner (2002) y Bagolin, Gabe y Ribeiro (2003), se hallaron pruebas de la curva de Kuznets para la región sur del Brasil. Kuznets (1966) argumenta que, independientemente del nivel inicial de desigualdad, el crecimiento económico puede conducir a la igualdad de ingresos a largo plazo. En tal sentido, el autor sostiene que en sus etapas iniciales la desigualdad sería pro crecimiento. La curva de Kuznets, en forma de "U" invertida, establece una fuerte evidencia empírica de que en las etapas intermedias de desarrollo la profundización de la desigualdad sería necesaria y un

\footnotetext{
${ }^{1}$ Las economías de escala dinámicas se generan mediante progreso tecnológico, ganancias de aprendizaje, economías externas y división del trabajo. El proceso de ganancias de aprendizaje puede mejorar la capacidad para la implementación de innovaciones.
}

fenómeno natural. Si bien a largo plazo predominarían las formas igualitarias, ello sería una consecuencia natural del proceso de crecimiento económico. Con este argumento se sostiene que el aumento del tamaño relativo de la industria conduciría al crecimiento de la productividad, que a su vez se traduciría en el incremento de la remuneración de los trabajadores especializados. La escasez de trabajadores calificados y de capital causaría el engrosamiento de la remuneración de la mano de obra en las etapas iniciales e intermedias de desarrollo. Con el tiempo, la oferta de trabajadores calificados se acrecentaría y, en consecuencia, su remuneración tendería a decrecer, reduciendo el grado de desigualdad de la economía.

Sin embargo, el estudio de la desigualdad en una base bidimensional es problemático. En ese sentido, se deben incorporar al análisis los argumentos teóricos en favor de la igualdad. Si la redistribución del ingreso mejora los niveles de educación y salud, esto producirá crecimiento económico, porque la productividad del trabajo se correlaciona positivamente con la educación y la salud a largo plazo. Si la relación entre ingreso absoluto y nivel de salud es cóncava, la redistribución del ingreso en beneficio de los trabajadores mejorará la salud de la población (Deaton, 2003) y, asimismo, ayudará a que esta supere la línea de pobreza. Por consiguiente, los efectos en la educación y el acceso a la salud estimularán el crecimiento económico a largo plazo. Además, en una economía basada en los salarios se puede alcanzar la prosperidad mediante políticas redistributivas.

En una economía en que los salarios tienen una alta incidencia en el crecimiento, la acumulación de capital está incentivada por las remuneraciones del trabajo (y el consumo). En ese caso, el efecto de la demanda 
(medido por el acelerador) sería un elemento esencial en la explicación de la acumulación, mientras que la participación de los beneficios en el producto sería una variable explicativa de menor importancia. Para los estructuralistas, el acelerador (efecto de la demanda) es clave en la explicación de la acumulación, ya que es superior al efecto de los beneficios en la inversión en la mayoría de los países en desarrollo (Taylor, 1983).

La redistribución en favor de los trabajadores puede traducirse en la expansión de la economía por medio de diversos canales, entre ellos la disminución de la inestabilidad política y de las restricciones al crédito. La reducción de la inestabilidad puede estimular la inversión productiva, lo que deriva en un aumento de la productividad, mientras que la merma de las restricciones al crédito puede incrementar la productividad a partir de la acumulación de conocimientos. Asimismo, la disminución de la inestabilidad puede fomentar reformas institucionales y la oferta de bienes públicos, que a su vez promueven un mayor grado de cohesión y facilitan el consenso con respecto a un proyecto común para la nación. La redistribución del ingreso también acrecienta el tamaño del mercado interno y produce de ese modo economías de escala considerables. Ros (2000) sugiere que la igualdad puede aminorar los incentivos para que los agentes económicos emprendan actividades de captación de rentas.

Un resultado similar puede alcanzarse mediante un análisis microeconómico. Según el enfoque de la utilidad, el abandono de la hipótesis de preferencias exógenas conduce a la conclusión de que la distribución del ingreso puede maximizar la utilidad agregada de la economía. Esto resulta evidente cuando se asume que la utilidad individual es una función del bienestar de otros individuos de la comunidad (Bortis, 1997).

De este modo, la redistribución del ingreso en favor de los trabajadores puede aumentar el nivel de utilidad agregada, pues la ganancia de utilidad de los segmentos de bajos y medios ingresos compensa la mengua de la utilidad de las franjas superiores. De lo contrario, la concentración del ingreso actuaría en forma perversa. Por lo menos desde el punto de vista teórico, la utilidad de un individuo se puede incrementar si otros ciudadanos reciben una cantidad mínima de bienes. En ese contexto, la redistribución puede justificarse si las preferencias son endógenas y están interrelacionadas.

Los argumentos anteriores denotan que la relación entre la desigualdad y la expansión de la actividad económica es bastante compleja. La desigualdad de ingresos puede derivar de distintos factores y es importante descubrir cuál de ellos predomina en su explicación. Si el mejoramiento de los niveles de educación y salud de la población compensa los efectos reductores del ahorro interno, la redistribución del ingreso será crucial para la expansión de la economía. Por consiguiente, es importante acceder a los efectos de la desigualdad en forma amplia, incluidas la desigualdad en la esfera política, de ingresos y en el acceso a la tierra. En la próxima sección se presentan las fuentes de datos y la metodología.

\section{IV}

\section{Fuentes de datos y procedimiento de Toda y Yamamoto (1995)}

En este trabajo se realizan pruebas de no causalidad de Granger para la función de inversión kaleckiana con respecto al Brasil en el período 1950-2008. La especificación de la función de inversión normalizada $(\mathrm{g}=\mathrm{I} / \mathrm{K})$, de acuerdo con las propuestas originales de Kalecki (1971) y Steindl (1952), incluye como variables independientes la participación de los beneficios en el producto (profit-share) $(\pi)$ y la capacidad utilizada $(u)$. Con respecto a $\pi$, esta se calculó como el cuociente entre los beneficios totales y el valor agregado. La capacidad utilizada se estimó mediante la razón entre el valor agregado y el capital nacional, según la literatura neokaleckiana. Los datos brutos empleados para el cálculo de la inversión, el capital nacional y la participación de los beneficios en el producto se tomaron de las estadísticas de IPEA (2011) y Marquetti (2000). Se empleó la base de datos del capital nacional extendida hasta 2008 de este último autor.

Las series son anuales y se utilizan como base los valores constantes de 1995. El método econométrico elegido es el procedimiento desarrollado por Toda y Yamamoto (1995) para probar la no causalidad de Granger. Todas las estimaciones se efectuaron con el programa. 
La prueba de no causalidad de Granger presupone que las variables son estacionarias. Las pruebas tradicionales no son adecuadas en presencia de variables integradas, puesto que no siguen una distribución estándar. Para superar ese problema, se aplican las pruebas de raíz unitaria y de cointegración.

No obstante, las pruebas econométricas de raíz unitaria (prueba de Dickey-Fuller y prueba de PhillipsPerron) son poco eficaces con respecto a la hipótesis alternativa de estacionariedad y no son confiables en caso de muestras relativamente pequeñas (Toda y Yamamoto, 1995, pág. 226). La prueba de cointegración (prueba de Johansen) presenta limitaciones similares, pues sus resultados son sensibles a las diferentes especificaciones adoptadas. Esto aumenta la incerteza de los resultados de las pruebas de causalidad, debido a la presencia de pruebas preliminares sesgadas (Marquetti, Koshiama y Alencastro, 2009, pág. 375).

El método de Toda y Yamamoto (1995) no presenta las limitaciones de los procedimientos tradicionales descritos anteriormente. Este método alternativo puede aplicarse a series cointegradas, no cointegradas o con diferente orden de integración, sin necesidad de proceder a las pruebas de raíz unitaria. Además, se trata de la prueba más indicada para muestras relativamente pequeñas (Marquetti, Koshiama y Alencastro, 2009, pág. 376; y Yamada y Toda, 1998).

El procedimiento desarrollado por Toda y Yamamoto (1995) consiste en la aplicación de una prueba de Wald para verificar las restricciones de los parámetros de un modelo de autorregresión vectorial (VAR), aumentado en niveles y estimado mediante mínimos cuadrados ordinarios (MCO). Toda y Yamamoto (1995) demostraron que la aplicación de la prueba de Wald para probar la restricción en los parámetros de un VAR $\left(\mathrm{k}+e_{\text {max }}\right)$ aumentado en niveles, sigue una distribución asintótica Chi-cuadrado $\left(\chi^{2}\right)$, independientemente de que el sistema sea cointegrado. $k$ es el número óptimo de rezagos y $e_{\max }$ el orden máximo de integración de las series temporales. Así, se aplica la prueba de Wald en los $k$ primeros parámetros con el fin de verificar la validez de la hipótesis de no causalidad de Granger. Los demás parámetros desfasados no se prueban, pues sirven únicamente para asegurar la presencia de una distribución asintótica Chi-cuadrado $\left(\chi^{2}\right)$.

La prueba elaborada por Toda y Yamamoto (1995) para probar la no causalidad de Granger incluye tres pasos. En primer lugar, se debe definir el número de rezagos $(k)$ y el orden máximo de integración del sistema $\left(e_{\text {max }}\right)$. En este artículo, como en la propuesta original de Toda y Yamamoto (1995), el número óptimo de rezagos se elegirá empleando el criterio de información de Schwartz (SIC $)^{2}$. Se determinó que el orden máximo de integración del sistema $\left(e_{\max }\right)$ sigue un proceso integrado de primer orden, pues la mayoría de las variables económicas son integradas de primer orden, I(1).

El paso siguiente consiste en la estimación directa de un $\operatorname{VAR}\left(\mathrm{k}+e_{\text {max }}\right)$ en niveles para las variables analizadas. A continuación se detallan las ecuaciones para la primera especificación:

$$
\begin{aligned}
& (g)_{a}=c_{1}+\alpha_{1 j} \sum_{j=1}^{k}(g)_{a j}+\delta_{1 j} \sum_{j=1}^{k}(\pi)_{a j}+\beta_{1 j} \sum_{j=1}^{k}(u)_{a j} \\
& +\alpha_{1 l} \sum_{l=k+1}^{e}(g)_{a}+\delta_{1 l} \sum_{l=k+1}^{e}(\pi)_{a}+\beta_{1 l} \sum_{l=k+1}^{k}(u)_{a}+\tau_{1 t} \\
& (\pi)_{a}=c_{2}+\alpha_{2 j} \sum_{j=1}^{k}(g)_{a j}+\delta_{2 j} \sum_{j=1}^{k}(\pi)_{a j}+\beta_{2 j} \sum_{j=1}^{k}(u)_{a j} \\
& +\alpha_{2 l} \sum_{l=k+1}^{e}(g)_{a}+\delta_{2 l} \sum_{l=k+1}^{e}(\pi)_{a}+\beta_{2 l} \sum_{l=k+1}^{k}(u)_{a}+\tau_{2 t} \\
& (u)_{a}=c_{3}+\alpha_{3 j} \sum_{j=1}^{k}(g)_{a j}+\delta_{3 j} \sum_{j=1}^{k}(\pi)_{a j}+\beta_{3 j} \sum_{j=1}^{k}(u)_{a j} \\
& +\alpha_{3 l} \sum_{l=k+1}^{e}(g)_{a}+\delta_{3 l} \sum_{l=k+1}^{e}(\pi)_{a}+\beta_{3 l} \sum_{l=k+1}^{k}(u)_{a}+\tau_{3 t}
\end{aligned}
$$

donde:

$\mathrm{g}=$ la tasa de acumulación (I/K);

$\pi=$ la participación de los beneficios en el producto;

$u=$ la capacidad utilizada $(\mathrm{Y} / \mathrm{K})$.

La última etapa consiste en la realización de la prueba de restricciones de Wald en los $k$ primeros parámetros, a fin de examinar la hipótesis de no causalidad de Granger. De esta manera, la participación de los beneficios en el producto causa, en el sentido de Granger, la tasa de acumulación si se rechaza la hipótesis $H_{0}: \delta_{1 j}=0$. Al mismo tiempo, la capacidad instalada $(u)$ causa, en el sentido de Granger, la acumulación si se rechaza la hipótesis $H_{0}: \beta_{1 j}=0$. Con respecto a las pruebas desarrolladas para determinar si la acumulación causa —en el sentido de Granger - tanto la participación de los beneficios en el producto, como la capacidad instalada, respectivamente, se procedió en forma análoga.

${ }^{2}$ Si bien se podría utilizar el criterio de información de Akaike (AIC) para definir el orden máximo de integración del sistema, su aplicación tiende a seleccionar modelos poco parsimoniosos. 
Así, el procedimiento desarrollado por Toda y Yamamoto (1995) con el fin de probar la hipótesis de no causalidad de Granger entre las variables, es un método adecuado para establecer las variables que afectarían a la tasa de acumulación nacional, y puede ofrecer elementos

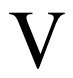

\section{Resultados}

En esta sección se analizan los principales resultados obtenidos. En primer lugar, se procede al análisis del orden de integración de las variables estudiadas. A continuación, se aplica el método de Toda y Yamamoto (1995) para probar la hipótesis de no causalidad de Granger entre las variables.

En el gráfico 3 se puede apreciar el comportamiento temporal de la acumulación de capital $(g)$, la participación de los beneficios en el producto $(\pi)$ y la capacidad utilizada $(u)$ en el período comprendido entre $1950 \mathrm{y}$ 2008. El análisis de este estudio se limitó a 2008, pues no existen estimaciones del capital nacional después de esa fecha. Como base para las referidas series se tomaron los precios de 1995, siendo la serie anual.

Se confirman las afirmaciones referidas anteriormente, sobre todo la relativa a la recuperación de la acumulación de capital y de la tasa de inversión a partir de 2003. para verificar si la economía está basada en los salarios o en las ganancias. Cabe subrayar que la prueba de no causalidad de Granger solo señala la precedencia temporal entre las variables de estudio y es un indicador en la previsión del comportamiento futuro de las variables.

Además, la razón entre los beneficios y el producto disminuye a partir de 2003 (Marquetti y Porsse, 2014), evidenciando el aumento de los salarios en el período.

En el cuadro 2 se muestran los resultados de las pruebas de raíz unitaria para las variables explicativas de la función de acumulación neokaleckiana. Se aplicó la prueba de Dickey-Fuller aumentada para verificar el orden de integración de las series.

Del análisis del cuadro 2 se desprende que posiblemente las variables relación beneficios-producto $(\pi)$ y capacidad utilizada $(u)$ tengan raíz unitaria. Sin embargo, los resultados no son concluyentes para esas dos variables, pues estas son sensibles a la especificación adoptada. Se observa también que la variable "acumulación de capital" tiene raíz unitaria. Se probó la primera diferencia de las variables, que indicó la estacionariedad de las series. inversión, participación de los beneficios en el producto $(\pi)$ y capacidad utilizada (u), 1950-2008
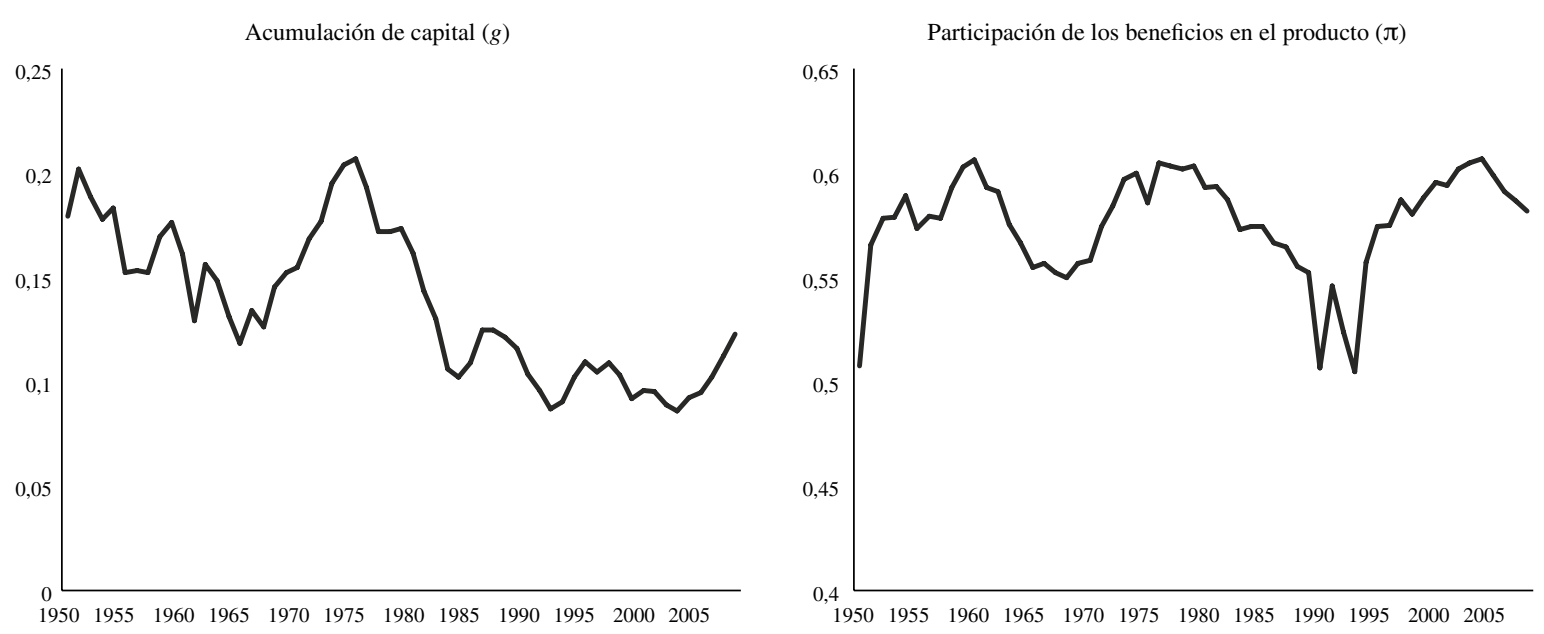

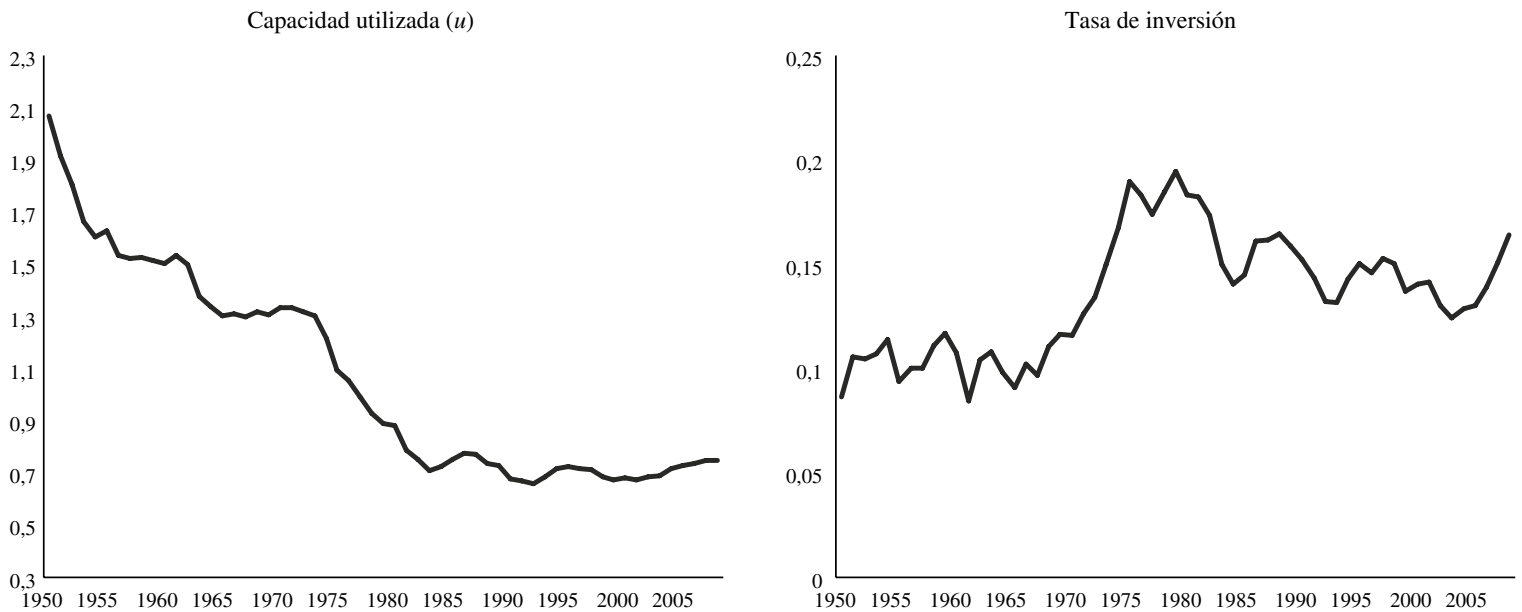

Fuente: Instituto Brasileño de Geografía y Estadística (IBGE), “Tabela de recursos e usos”, 2011 [en línea] http://www.ibge.gov.br/home/ estatistica/economia/contasnacionais/2011; Instituto de Investigación Económica Aplicada (IPEA), "Estatísticas sociais”, Ipeadata, 2011 [en línea] http://www.ipeadata.gov.br, y A.A. Marquetti, "Estimativa do estoque de riqueza tangível no Brasil, 1950-1998", Nova Economia, vol. 10, $\mathrm{N}^{\circ}$ 2, Minas Gerais, Universidad Federal de Minas Gerais (UFMG), 2000.

Nota: además, se utilizó la base de datos de capital nacional de Marquetti (2000) extendida hasta 2008 y Marquetti y Porsse (2014). La acumulación de capital se estimó mediante la razón entre la inversión y el capital nacional. La capacidad utilizada resultó de la división entre el producto por el capital nacional, en conformidad con el enfoque neokaleckiano. Por último, la tasa de inversión es simplemente la razón entre la inversión y el producto.

CUADRO 2

Prueba de raíz unitaria para las variables de la regresión

\begin{tabular}{|c|c|c|c|c|}
\hline Variables & Modalidad de la prueba & $\begin{array}{l}\text { Número de } \\
\text { rezagos }\end{array}$ & $\begin{array}{c}\text { Prueba de Dickey-Fuller } \\
\text { aumentada }\end{array}$ & Valor $p$ \\
\hline \multirow{3}{*}{ Acumulación de capital $(g)$} & sin constante & 1 & $-0,95$ & 0,30 \\
\hline & con constante & 1 & $-1,59$ & 0,47 \\
\hline & con constante y tendencia & 1 & $-2,25$ & 0,45 \\
\hline \multirow[t]{3}{*}{ Relación beneficios-producto $(\pi)$} & sin constante & 1 & 0,47 & 0,81 \\
\hline & con constante & 3 & $-2,93$ & $0,04 * *$ \\
\hline & con constante y tendencia & 3 & $-2,90$ & 0,16 \\
\hline \multirow[t]{3}{*}{ Capacidad instalada $(u)$} & sin constante & 1 & $-2,48$ & $0,01 * * *$ \\
\hline & con constante & 1 & $-2,22$ & 0,20 \\
\hline & con constante y tendencia & 1 & $-1,29$ & 0,87 \\
\hline
\end{tabular}

Fuente: elaboración propia.

Se aplicó el procedimiento de Toda y Yamamoto (1995) en la función de acumulación de capital neokaleckiana para establecer si las series presentan causalidad unidireccional o bidireccional en el sentido de Granger. En la primera etapa de la prueba se debe definir el nivel del modelo de autorregresión vectorial VAR aumentado, es decir, definir el número de rezagos $(k)$ y el orden máximo de integración del sistema $\left(e_{\text {max }}\right)$.

Al proseguir con el análisis del cuadro 2 se observa que el orden máximo de integración del sistema $\left(e_{\max }\right)$ es 1. El número de rezagos $(k)$ se obtuvo mediante la prueba de Scharwtz y resultó igual a 2. A continuación, se estimaron un $\operatorname{VAR}(3)$ en nivel para proceder con la prueba de no causalidad de Granger y un $\operatorname{vaR}(4)^{3}$ a objeto de verificar la sensibilidad de los resultados al nivel de rezagos del sistema.

\footnotetext{
${ }^{3}$ Se realizaron pruebas para comprobar la existencia de autocorrelación de los residuos: prueba LM y prueba de heteroscedasticidad de White en la especificación de la función de inversión en forma intensiva.
} 
Los resultados para la especificación neokaleckiana sugieren que la capacidad utilizada $(u)$ precede temporalmente a la acumulación de capital. O sea, la capacidad instalada ${ }^{4}$ (cuyo coeficiente en la ecuación es una medida del acelerador) causa, en el sentido de Granger, la acumulación. Cabe subrayar que los resultados no son sensibles al número de rezagos elegidos en la prueba de Granger (véanse más detalles en el cuadro 3 $y$ en el anexo).

Del análisis del cuadro 3 surge que la relación beneficios-producto no causa, en el sentido de Granger, la acumulación de capital. Este resultado, junto con la indicación de que la capacidad utilizada $(u)$ causa, en el sentido de Granger, la acumulación (que ratifica el papel central del acelerador en las economías emergentes), es un indicador de que los procesos de concentración

\footnotetext{
${ }^{4}$ En general, existe una controversia entre marxistas y neokaleckianos sobre el empleo de la relación producto-capital como sustituto de la capacidad instalada de la economía. Para los marxistas, la productividad del capital (relación producto-capital) no sería una medida adecuada de la capacidad instalada (Duménil y Lévy, 1999). La productividad del capital sería solo el componente tecnológico de la tasa de beneficios $(\mathrm{r}=(\pi / \mathrm{Y})(\mathrm{Y} / \mathrm{K}))$, al ser una medida del cambio técnico de la economía y resultar del conflicto distributivo. Para los marxistas, el crecimiento del milagro económico brasileño (1968-1973) obedecería al cambio técnico y al alto nivel de la productividad del capital en el período.
}

y desconcentración del ingreso no determinarían necesariamente el crecimiento de la economía brasileña en el período 1950-2008. Por consiguiente, la economía podría crecer con la aplicación de políticas redistributivas o de concentración del ingreso, mientras que el arreglo institucional es un importante componente explicativo de la expansión.

En este contexto es importante retomar las ideas de Tavares y Serra (1972), quienes sugieren que el agotamiento del modelo de crecimiento brasileño de la década de 1960 se debió a la merma de la inversión. Esos autores afirman que la economía demandaría arreglos que garantizaran nuevas fuentes de financiamiento para aumentar la inversión. De ahí la necesidad de reconcentrar el ingreso como mecanismo orientado a proveer nuevas fuentes de recursos que incrementen la acumulación. En suma, Tavares y Serra (1972) creen que la parálisis o el agotamiento de los regímenes de crecimiento están relacionados con la propia dinámica del sistema, que crea restricciones que exigen nuevos arreglos sociales, económicos y políticos.

Por último, se verifica que no existe relación de causalidad, en el sentido de Granger, entre la participación de los beneficios en el producto y la capacidad utilizada. En otras palabras, no existe precedencia temporal entre las dos series.

CUADRO 3

Brasil: relaciones de causalidad de Granger en la economía, 1950-2008

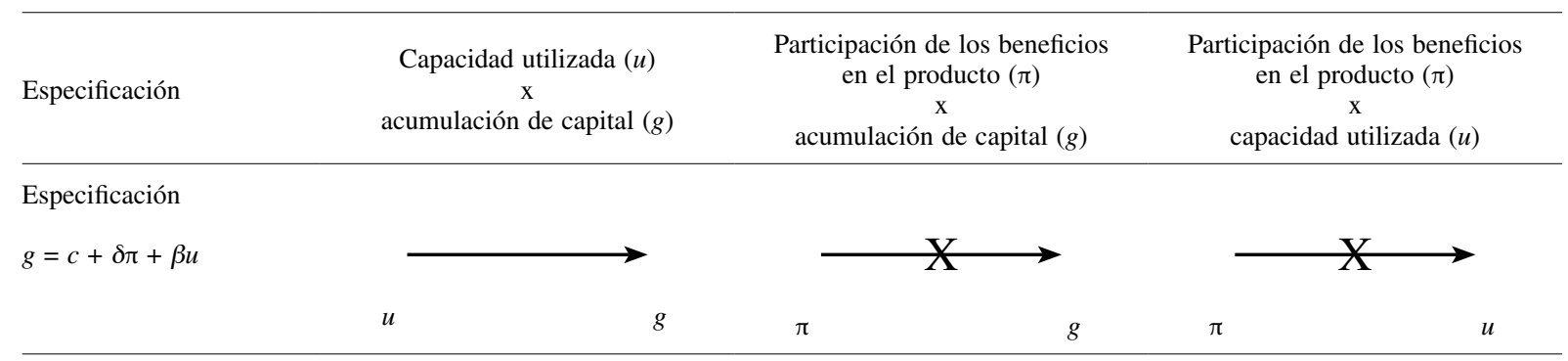

Fuente: elaboración propia. 


\section{VI}

\section{Conclusiones}

El objetivo de este artículo era establecer si existe causalidad, en el sentido de Granger, entre las variables: participación de los beneficios en el producto, capacidad utilizada y acumulación de capital. Los resultados de las pruebas de no causalidad de Granger para las variables de la función de inversión en forma intensiva proporcionan un indicador del régimen de crecimiento de la economía brasileña en el período 1950-2008.

Los resultados de las pruebas revelan que solo la capacidad utilizada causa, en el sentido de Granger, la acumulación de capital. Se observó también que la participación de los beneficios en el producto no causa, en el sentido de Granger, la acumulación. En ese sentido, la economía puede presentar regímenes de crecimiento basados en los salarios y en las ganancias en determinados períodos. Los regímenes basados en los salarios y en las ganancias explicarían los procesos de expansión solo a corto y mediano plazo (Taylor, 1991)

En consecuencia, estos resultados indican que el crecimiento de la economía del Brasil se explicaría por el acelerador.

Específicamente, los resultados muestran que el acelerador (el coeficiente de la variable capacidad instalada en la regresión) es el parámetro clave para explicar la acumulación de capital brasileña. De acuerdo con la literatura neokaleckiana y estructuralista, el acelerador es el principal componente explicativo de la inversión, al ser superior al efecto de los beneficios en la inversión en los países en desarrollo (Taylor, 1983). En otros estudios, como por ejemplo los de Cuesta (1990) y Von Arnim y Rada (2011), se obtuvieron resultados similares utilizando métodos alternativos para el estudio de otros países.

De esta forma, los resultados indican que el acelerador aumentaría la acumulación de capital, incrementando el nivel de actividad económica. De acuerdo con las estimaciones obtenidas, el crecimiento se produciría a través de la concentración o desconcentración del ingreso. En las economías se alternarían períodos de expansión impulsada por los salarios con períodos determinados por las ganancias, al tratarse de un fenómeno de corto o mediano plazo. Los factores principales serían el efecto acelerador y el arreglo institucional necesario para estimular la expansión. Los regímenes de crecimiento cambian a lo largo del tiempo, por lo que se sugiere proceder con cautela y profundizar las investigaciones para determinar el régimen actual de la economía brasileña.

ANEXO

CUADRO A.

Regresión del vector autorregresivo (VAR(3)), mínimos cuadrados ordinarios (MCO), 1950-2008

(Variable dependiente: acumulación de capital $(g)$ )

\begin{tabular}{|c|c|c|c|c|}
\hline Variable & Coeficiente & Error estándar & Estadística $t$ & Valor $p$ \\
\hline Constante & 0,039638 & 0,037287 & 1,063054 & 0,2933 \\
\hline$g(-1)$ & 0,954799 & 0,183519 & 5,202738 & 0,0000 \\
\hline$u(-1)$ & 0,095541 & 0,048361 & 1,975580 & 0,0542 \\
\hline$g(-2)$ & $-0,093285$ & 0,257331 & $-0,362509$ & 0,7186 \\
\hline$\pi(-2)$ & 0,095327 & 0,074971 & 1,271519 & 0,2099 \\
\hline$u(-2)$ & 0,008393 & 0,070331 & 0,119337 & 0,9055 \\
\hline$g(-3)$ & 0,105512 & 0,132798 & 0,794533 & 0,4310 \\
\hline$\pi(-3)$ & $-0,081704$ & 0,078005 & $-1,047412$ & 0,3004 \\
\hline$u(-3)$ & $-0,093466$ & 0,054570 & $-1,712765$ & 0,0935 \\
\hline R-cuadrado & 0,908229 & \multicolumn{2}{|c|}{ R-cuadrado ajustado } & 0,890274 \\
\hline S.E. de la regresión & 0,011322 & \multicolumn{2}{|c|}{ Criterio de Akaike } & $-5,963656$ \\
\hline Suma cuadrado de los residuos & 0,005897 & \multicolumn{2}{|c|}{ Criterio de Schwarz } & $-5,601986$ \\
\hline Durbin-Watson & 1,958439 & \multicolumn{2}{|c|}{ Prob. (estadística F) } & 0,000000 \\
\hline
\end{tabular}

Fuente: elaboración propia.

Nota: las variables de la regresión son: acumulación de capital $(g)$, participación de los beneficios en el producto $(\pi)$ y capacidad utilizada $(u)$. 
CUADRO A.2

Prueba de Wald para la hipótesis de que la variable participación de los beneficios en el producto $(\pi)$ causa, en el sentido de Granger, la acumulación brasileña de capital $(g)$

\begin{tabular}{lccc}
\hline Prueba estadística & Valor & Grados de libertad & Valor $p$ \\
\hline Estadística F & 0,904804 & $(2,46)$ & 0,4117 \\
Chi-cuadrado & 1,809608 & 2 & 0,4046 \\
\hline
\end{tabular}

Fuente: elaboración propia.

CUADRO A.3

Prueba de Wald para la hipótesis de que la variable capacidad utilizada (u) causa, en el sentido de Granger, la acumulación de capital brasileña $(g)$

\begin{tabular}{|c|c|c|c|}
\hline Prueba estadística & Valor & Grados de libertad & Valor $p$ \\
\hline Estadística F & 3,372944 & $(2,46)$ & 0,0430 \\
\hline Chi-cuadrado & 6,745888 & 2 & 0,0343 \\
\hline
\end{tabular}

Fuente: elaboración propia.

CUADRO A.4

Regresión del vector autorregresivo (VAR(4)), mínimos cuadrados ordinarios (MCO), 1950-2008

(Variable dependiente: acumulación de capital $(g)$ )

\begin{tabular}{|c|c|c|c|c|}
\hline Variable & Coeficiente & Error estándar & Estadística $t$ & Valor $p$ \\
\hline Constante & 0,044792 & 0,035378 & 1,266118 & 0,2124 \\
\hline$g(-1)$ & 0,968530 & 0,214332 & 4,518826 & 0,0000 \\
\hline$\pi(-1)$ & $-0,100200$ & 0,092328 & $-1,085258$ & 0,2840 \\
\hline$u(-1)$ & 0,103160 & 0,047612 & 2,166659 & 0,0360 \\
\hline$g(-2)$ & $-0,072161$ & 0,248043 & $-0,290920$ & 0,7725 \\
\hline$\pi(-2)$ & 0,096273 & 0,076167 & 1,263974 & 0,2132 \\
\hline$u(-2)$ & 0,008107 & 0,074341 & 0,109053 & 0,9137 \\
\hline$g(-3)$ & 0,047919 & 0,182107 & 0,263136 & 0,7937 \\
\hline$\pi(-3)$ & 0,015815 & 0,105128 & 0,150432 & 0,8811 \\
\hline$u(-3)$ & $-0,127061$ & 0,098793 & $-1,286133$ & 0,2054 \\
\hline$g(-4)$ & 0,020818 & 0,150357 & 0,138454 & 0,8905 \\
\hline$\pi(-4)$ & $-0,094231$ & 0,076204 & $-1,236561$ & 0,2231 \\
\hline$u(-4)$ & 0,025055 & 0,068363 & 0,366495 & 0,7158 \\
\hline R-cuadrado & 0,907611 & \multicolumn{2}{|c|}{ R-cuadrado ajustado } & 0,881214 \\
\hline S.E. de la regresión & 0,011708 & \multicolumn{2}{|c|}{ Criterio de Akaike } & $-5,854008$ \\
\hline Suma cuadrado de los residuos & 0,005757 & \multicolumn{2}{|c|}{ Criterio de Schwarz } & $-5,379548$ \\
\hline Durbin-Watson & 1,954304 & \multicolumn{2}{|c|}{ Prob. (estadística F) } & 0,00000 \\
\hline
\end{tabular}

Fuente: elaboración propia.

Nota: las variables de la regresión son: acumulación de capital $(g)$, participación de los beneficios en el producto $(\pi)$ y capacidad utilizada $(u)$.

CUADRO A.5

Prueba de Wald para la hipótesis de que la variable participación de los beneficios en el producto $(\pi)$ causa, en el sentido de Granger, la acumulación brasileña de capital (g)

\begin{tabular}{|c|c|c|c|}
\hline Prueba estadística & Valor & Grados de libertad & Valor $p$ \\
\hline Estadística F & 1,103795 & $(2,42)$ & 0,3410 \\
\hline Chi-cuadrado & 2,207590 & 2 & 0,3316 \\
\hline
\end{tabular}

Fuente: elaboración propia. 
Prueba de Wald para la hipótesis de que la variable capacidad utilizada (u) causa, en el sentido de Granger, la acumulación de capital brasileña $(g)$

\begin{tabular}{|c|c|c|c|}
\hline Prueba estadística & Valor & Grados de libertad & Valor $p$ \\
\hline Estadística F & 3,484320 & $(2,42)$ & 0,0398 \\
\hline Chi-cuadrado & 6,968640 & 2 & 0,0307 \\
\hline
\end{tabular}

Fuente: elaboración propia.

Se encontraron los mismos resultados para la causalidad de Granger en el VAR(4), de modo que no son sensibles al número de rezagos del sistema. En forma análoga, se estimó un VAR en niveles para verificar si las variables causan, en el sentido de Granger, la capacidad utilizada y la participación de los beneficios en el producto. Los resultados indican que la acumulación de capital no causa, en el sentido de Granger, la capacidad utilizada y la relación beneficios-producto. Se puede acceder a los resultados completos de estas pruebas mediante solicitud a los autores.

\section{Bibliografía}

Badhuri, A. y S. Marglin (1990), "Unemployment and real wage: The economic basis for contesting political ideologies", Cambridge Journal of Economics, vol. 14, $\mathrm{N}^{\circ} 4$, Oxford, Oxford University Press.

Bagolin, I.P., J. Gabe y E.P. Ribeiro (2003), "Crescimento e desigualdade no Rio Grande do Sul: uma revisão da Curva de Kuznets para os municípios gaúchos (1970-1991)”, inédito.

Berni, D.A., A.A. Marquetti y R. Kloeckner (2002), "A desigualdade econômica do Rio Grande do Sul: primeiras investigações sobre a Curva de Kuznets", Anais do $1^{\circ}$ Encontro de Economia Gaúcha, Porto Alegre.

Bortis, H. (1997), Institutions, Behaviour and Economic Theory: A Contribution to Classical-Keynesian Political Economy, Cambridge, Cambridge University Press.

CEPAL (Comisión Económica para América Latina y el Caribe) (2012), "CEPALSTAT" [en línea] http://estadisticas.cepal.org/ cepalstat/WEB_CEPALSTAT/Portada.asp.

Cuesta, J.L.L. (1990), "IS-FM macroeconomics: general equilibrium linkages of the food market in Colombia", Socially Relevant Policy Analysis: Structuralist Computable General Equilibrium Models for the Developing World, L. Taylor (ed.), Cambridge, Massachusetts, The MIT Press.

Deaton, A. (2003), "Health, inequality, and economic development", Journal of Economic Literature, vol. 41, $\mathrm{N}^{\circ} 1$, Nashville, Tennessee, American Economic Association.

Duménil, G. y D. Lévy (1999), "Being Keynesian in the short term and classical in the long term: The traverse to classical long-term equilibrium", The Manchester School, vol. 67, $\mathrm{N}^{\circ}$ 6, Wiley.

Dutt, A.K. (1984), "Stagnation, income distribution and monopoly power", Cambridge Journal of Economics, vol. 8, № 1, Oxford, Oxford University Press.

Furtado, C. (1972), Análise do modelo brasileiro, Río de Janeiro, Civilização Brasileira.

(1965), Subdesenvolvimento e estagnação na América Latina, Río de Janeiro, Civilização Brasileira.

IBGE (Instituto Brasileño de Geografía y Estadística) (2011), "Tabela de recursos e usos" [en línea] http://www.ibge.gov.br/home/ estatistica/economia/contasnacionais/2011.

IPEA (Instituto de Investigación Económica Aplicada) (2011), "Estatísticas sociais", Ipeadata [en línea] http://www.ipeadata. gov.br.

Kalecki, M. (1971), Selected Essays on the Dynamics of the Capitalist Economy, Cambridge, Cambridge University Press.

Kuznets, S. (1966), Modern Economic Growth, New Haven, Yale University Press.
Marquetti, A.A. (2000), "Estimativa do estoque de riqueza tangível no Brasil, 1950-1998", Nova Economia, vol. 10, № 2, Minas Gerais, Universidad Federal de Minas Gerais (UFMG).

Marquetti, A.A., D. Koshiama y D. Alencastro (2009), "O aumento da lucratividade expande a acumulação de capital? Uma análise de causalidade de Granger para países da OCDE", Revista de Economia Contemporânea, vol. 13, N 3, Río de Janeiro, Instituto de Economía, Universidad Federal de Río de Janeiro.

Marquetti, A.A. y M.C.S. Porsse (2014), "Patrones de progreso técnico en la economía brasileña, 1952-2008”, Revista CEPAL, $\mathrm{N}^{\circ} 113$, Santiago de Chile, Comisión Económica para América Latina y el Caribe.

Naciones Unidas (2010), Situación y perspectivas de la economía mundial 2010, Nueva York.

oIT (Organización Internacional del Trabajo) (2011), Studies on Growth with Equity. Brazil: An Innovative Income-led Strategy, Ginebra, Instituto Internacional de Estudios Laborales.

Ranis, G. y F. Stewart (2000), "Strategies for success in human development", Journal of Human Development, vol. 1, $\mathrm{N}^{\circ} 1$, Taylor \& Francis.

Ros, J. (2000), Development and the Economics of Growth, Ann Arbor, The University of Michigan Press.

Steindl, J. (1952), Maturity and Stagnation in American Capitalism, Oxford, Basil Blackwell.

Tavares, C. y J. Serra (1972), "Além da estagnação", Da substituição de importações ao capitalismo financeiro, M.C. Tavares, Río de Janeiro, Zahar Editores.

Taylor, L. (1991), Income Distribution, Inflation, and Growth: Lectures on Structuralist Macroeconomic Theory, Cambridge, Massachusetts, The MIT Press.

(1983), Structuralist Macroeconomics: Applicable Models for the Third World, Nueva York, Basic Books.

Toda, H. y T. Yamamoto (1995), "Statistical inference in vector autoregressions with possibly integrated processes", Journal of Econometrics, vol. 66, $\mathrm{N}^{\circ} 1-2$, Amsterdam, Elsevier.

Verbeek, M. (2008), A Guide to Modern Econometrics, John Wiley \& Sons Ltd.

Von Arnim, R. y C. Rada (2011), "Labour productivity and energy use in a three-sector model: An application to Egypt", Development and Change, vol. 42, $\mathrm{N}^{\circ}$ 6, Wiley.

Yamada, H. y H. Toda (1998), "Inference in possibly integrated vector autorregresive models: Some finite sample evidence", Journal of Econometrics, vol. 86, $\mathrm{N}^{\circ} 1$, Amsterdam, Elsevier. 